\title{
Prophylaxis of migraine headache
}

\author{
Tamara Pringsheim MD MSc, W. Jeptha Davenport BA MD, Werner J. Becker MD
}

Previously published at www.cmaj.ca

\section{The case}

A 40-year-old woman presented with an eight-year history of migraine headaches. The headaches occurred once or twice per week, but usually responded well to a triptan taken soon after onset. Sometimes the headache returned the next day, necessitating a second dose. Occasionally the headaches did not respond well to the triptan; they caused the patient to miss work about once per month. The patient's headache diary shows that she takes a triptan nine days per month. Her physician suggests a prophylactic medication, given that she is at risk for medication overuse headache and experiencing substantial disability despite taking the medication. The patient's medical history includes asthma that is well controlled. She generally sleeps well and has no history of clinically significant mood disturbance. Physical examination is normal except for obesity. Which prophylactic medication should be tried first?

$\mathrm{M}$ igraine headache is a common, disabling condition. The diagnostic criteria for migraine headache ${ }^{1}$ according to the International Headache Society are shown in Box 1. When migraine episodes are frequent, treatment can be challenging. Prophylactic therapy for migraine remains one of the more difficult aspects. Although valid randomized controlled trials exist to aid decisionmaking, all of the medications used in treatment have incomplete efficacy, and most produce adverse effects.

In this review, we discuss when to consider prophylaxis for the patient with migraine, and provide a systematic review of the evidence available from randomized controlled trials on prophylactic agents. Existing guidelines on this topic are out of date ${ }^{2}$ or do not include a systematic review of the literature. ${ }^{3}$

Medications used to treat this condition can be divided into two broad categories: symptomatic or acute-care medications to treat individual migraine episodes, and prophylactic or preventive medications, which are used to reduce headache frequency.

Symptomatic migraine therapy alone, although helpful for many patients, is not adequate treatment for all. Patients who have frequent episodes of migraine may sustain substantial disability despite appropriate symptomatic therapy. When symptomatic medications are used too frequently, they can result in increased frequency of headache and medication overuse headache. ${ }^{1,4}$ The Canadian Headache Outpatient Registry and Database Project found that $21 \%$ of patients with a

\section{Key points}

- The choice of prophylactic therapy for migraine is based on existing comorbidities, contraindications, reported efficacy, and adverse effect profiles.

- Prophylaxis should be considered for patients whose migraines affect their quality of life despite appropriate symptomatic treatment, or who are at risk for medication overuse headache.

- The period that successful migraine prophylaxis should be continued is not clear; patients should be followed up reg ularly to ensure that their medication is still beneficial.

diagnosis of migraine who were referred to headache specialists had symptomatic medication overuse. ${ }^{5}$

When prophylaxis should be started is a matter of clinical judgement. Evidence on which to base this decision is lacking, and many medications are available from which to choose. Prophylactic medications are no substitute for attention to patient lifestyle and avoidance of migraine triggers. All patients for whom migraine prophylactic agents are considered should be educated in migraine triggers and lifestylerelated factors. Common headache triggers include caffeine withdrawal, alcohol, sunlight, menstruation and changes in barometric pressure. Lifestyle factors, including stress, mealskipping, obesity, and erratic sleep and work schedules, can precipitate migraine.

\section{When should prophylaxis be considered?}

Prophylactic therapy should be considered for patients whose migraine episodes have a substantial impact on their lives (i.e., causing them to miss workdays and family activities) despite appropriate use of symptomatic medications, or whose frequency of episodes is such that reliance on acute medications alone puts them at risk for medication overuse headache. ${ }^{3}$ According to the criteria of the International Headache Society, ${ }^{1}$ medication overuse headache is defined as headache present on more than 15 days per month, which has developed or markedly worsened during medication overuse. The headache associated with medication overuse is

From the Calgary Headache Assessment and Management Program, Foothills Medical Centre, Department of Clinical Neurosciences, University of Calgary, Calgary, Alta.

CMAJ 2010. DOI:10.1503/cmaj.081657 


\section{Box 1: International Headache Society criteria for} migraine headache

A. At least five episodes fulfilling criteria B-D have occurred

B. Headaches last 4-72 hours (untreated or unsuccessfully treated)

C. Headache has at least two of the following characteristics:

Unilateral location

Pulsating quality

Moderate or severe pain intensity

Aggravation by or causing avoidance of routine physical activity (e.g., walking or climbing stairs)

D. During headache, at least one of the following is present:

- Nausea or vomiting, or both

Photophobia and phonophobia

E. Headache is not attributable to another disorder

variable, with features of both migraine and tension-type headache (i.e., bilateral location, pain that ranges from mild to severe, and associated nausea and light sensitivity). Medication overuse occurs when acute treatments are used frequently and regularly, i.e., 15 days or more per month for simple analgesics and 10 days or more per month for triptans, opioids, and combination analgesics.

Some guidelines have made consensus-based recommendations that prophylactic treatment should be considered when a patient has three or more severe migraine episodes per month that fail to respond adequately to symptomatic treatment with medication. ${ }^{6}$ Although episode frequency is helpful in determining the need for prophylactic therapy, the decision to discuss prophylaxis with the patient should be individualized, and all aspects of the patient's migraine syndrome, including the risk of symptomatic medication overuse, need to be considered. According to estimates, about $25 \%$ of all migraine sufferers should be offered prophylactic therapy, and prophylactic medications are thought to be underutilized. ${ }^{5}$

When migraine prophylactic therapy is started, one of three outcomes can be anticipated. The patient may show substantial benefit, which is usually defined as a reduction in frequency of headaches of $50 \%$ or more. The frequency can be assessed by having the patient keep a diary of the headaches. Although the primary effect of prophylactic medications is a reduction in frequency, some patients report that they also experience reduced intensity of their headaches and improved response to symptomatic medication while taking a prophylactic medication. ${ }^{8}$

The second possibility is that the patient may develop intolerable adverse effects (i.e., somnolence, nausea, cognitive slowing or weight gain), that may necessitate stopping therapy. The third possible outcome is that the medication may show insufficient efficacy. Most prophylactic medications show substantial efficacy within one month after initiation of treatment, and their therapeutic effect may continue to increase for several months after that point. ${ }^{9-11}$ If the patient shows no benefit after two months of therapy at the target dose, prophylactic treatment should be stopped and, if indicated, another medication tried.
Overuse of symptomatic medications for headache is considered by headache specialists to reduce the effectiveness of migraine prophylaxis. Avoidance of medication overuse is recommended to improve the likelihood of success when starting prophylactic therapy. ${ }^{1,6}$ Several clinical trials suggest that one particular prophylactic medication, topiramate, can be effective in reducing the frequency of migraine in the presence of medication overuse, and results in a reduction in use of symptomatic medication..$^{12,13}$

Two and a half per cent of migraine patients per year progress to a very frequent headache pattern, either as a result of medication overuse, or because of an apparent progression to chronic migraine ${ }^{14}$ Chronic migraine is defined as migraine that occurs on 15 or more days per month for more than three months. Patients with an intermediate headache frequency of six to nine days per month are at greater risk for further progression to chronic migraine. The risk is even greater in patients who have headaches on 10 to 14 days per month. ${ }^{15}$ Reduction in frequency of migraine through use of prophylactic therapy may prevent or delay progression to chronic migraine, although this effect is not proven. ${ }^{16}$

\section{Methods}

We performed a systematic review of prospective doubleblind, randomized controlled trials of medications for prevention of migraine episodes. Study participants in the trials were eligible if they were adults and met International Headache Society ${ }^{1}$ or Ad $\mathrm{Hoc}^{17}$ criteria for the diagnosis of migraine headache. We excluded trials of patients with chronic daily headache, chronic tension-type headache or chronic migraine. Because of the large number of different pharmacologic agents assessed over the past 60 years for the prophylaxis of migraine, we limited our search to agents that are commonly used in clinical practice in North America and Europe.

We searched Ovid MEDLINE (1950 to April 2008) and EMBASE (1980 to April 2008) using a search strategy that combined the subject search with a highly sensitive search string for randomized controlled trials. The subject search string used a combination of controlled vocabulary and freetext terms. In addition, we searched the Cochrane Collaboration Library for systematic reviews of agents used for migraine prophylaxis. We used Cochrane systematic reviews to summarize trial data if similar inclusion criteria and methodology were used in the review. The titles and abstracts of studies identified by the literature search were screened for eligibility by two independent reviewers (TP and WB). Papers that we could not exclude with certainty on the basis of the information contained in the title or abstract were retrieved in full for screening. Papers that passed the initial screening process were retrieved and the full text was reviewed independently by two reviewers (TP and WJD). We performed meta-analyses of selected groups of studies.

Our search yielded 883 abstracts and three Cochrane systematic reviews. After examination of the abstracts and systematic reviews, only 59 studies and one Cochrane systematic review met our inclusion criteria and were included. Most of the studies used the International Headache Society ${ }^{1}$ or $\mathrm{Ad}$ 
$\mathrm{Hoc}^{17}$ criteria for the diagnosis of migraine. Most participants were women. Pregnant or lactating women were excluded. Most studies specifically excluded people who had chronic daily headache or used medication on more than 10 to 15 days per month. To be included, participants in most of the studies had to have experienced between two and eight migraine episodes per month and not have taken other prophylactic treatment for migraine during the study period. The use of symptomatic therapy for migraine episodes was permitted.

The most consistently reported outcome among the studies was the responder rate, which is defined as the percentage of patients with a $50 \%$ or greater reduction in frequency of migraine compared with baseline. The duration of trials varied from 8 to 26 weeks, with most trials using a four-week prospective baseline followed by a 12 -week treatment phase.

\section{Pharmacologic prophylactic therapies}

The results of our review of studies for quality of evidence, impression of efficacy and frequency of adverse effects of medications for prophylaxis of migraine are summarized in Table 1. We used the criteria of the United States Preventive Task Force to grade the quality of evidence. ${ }^{76}$ The grading of the evidence may not be consistent with other guidelines or reviews that use different rating systems. An informational aid to choosing prophylactic therapy is provided in Table 2. Its guidance is based on expert opinion and analysis of the evidence from randomized controlled trials on treatment efficacy and adverse effects.

\section{Antiepileptics}

Our meta-analysis of three trials of the effectiveness of divalproex sodium ${ }^{18-20}$ (at dosages ranging from 500 to $1500 \mathrm{mg}$ ) involving 510 patients showed an odds ratio (OR) of 2.74 (95\% CI $1.48-5.08, p=0.001)$ for experiencing a $50 \%$ or greater reduction in frequency of migraines compared to placebo therapy. Adverse effects were greater in the treatment group receiving divalproex sodium, especially at higher doses, and included nausea, somnolence, tremor and dizziness (Table 1).

When divalproex sodium is prescribed to women of childbearing potential, it should be given with folic acid, and patients' birth-control status should be considered, given the potential for teratogenicity. Prospective data from the North American Antiepileptic Drug Pregnancy Registry show that major birth defects occurred in $10.7 \%$ of infants exposed to divalproex sodium in the first trimester, compared with $1.6 \%$ in external control infants (relative risk [RR] 7.3, 95\% CI 4.4-12.2). ${ }^{77}$ For this reason, many physicians choose to avoid this medication in this population of patients.

The odds ratio of a 50\% or greater reduction in frequency of migraine episodes using gabapentin ${ }^{28}$ compared to placebo was 4.51 (95\% CI 1.51-13.43, $p=0.007$ ), according to one trial of 143 migraine patients. The most common adverse effects of gabapentin are somnolence and dizziness.

Our meta-analysis of four trials comparing topiramate 100 $\mathrm{mg}$ to placebo ${ }^{11,22-25}$ in 828 patients showed an odds ratio of $2.44(95 \%$ CI $1.81-3.28, p<0.0001)$ for a $50 \%$ or greater reduction in migraine frequency relative to placebo. Although topiramate is very effective, adverse effects cause cessation of therapy in up to $30 \%$ of patients on the 200-mg dosage in randomized trials. Special considerations when prescribing topiramate include its interaction with the birth control pill (decreasing effectiveness of contraception) and potential to cause renal calculi and, rarely, acute angle closure glaucoma (i.e., shown in case reports only). ${ }^{78}$

Given the high number of adverse events and withdrawals on the 200-mg dosage of topiramate, the recommended dosage is $100 \mathrm{mg}$ per day. Topiramate may be useful for prevention of migraine in patients who are overweight because it does not cause weight gain and may help in weight loss. In head-tohead trials, topiramate appeared to have similar efficacy to propranolol ${ }^{22}$ and divalproex sodium. ${ }^{21}$ Patients with comorbid epilepsy or bipolar disorder may be offered an antiepileptic for migraine prophylaxis to simplify medication regimens, although no evidence exists to support this recommendation.

\section{Antidepressants}

Four trials evaluated amitriptyline $e^{30-33}$ as prophylaxis for migraine, and all reported a beneficial effect. Reports from clinical trials of amitriptyline for migraine prevention are generally of poor quality, and are heterogeneous with respect to design and outcomes, precluding meta-analysis. One trial ${ }^{31}$ of 100 patients found an odds ratio of 2.41 (95\% CI 1.07-5.40, $p$ $=0.03$ ) relative to placebo for a $50 \%$ or greater improvement in migraine. In all of the trials, the main adverse effects observed were dry mouth and drowsiness. Many headache specialists consider amitriptyline a first-line treatment. ${ }^{1,2}$ Amitriptyline reduces sleep latency, making the medication a good option for patients with coexisting initial insomnia.

Trials of venlafaxine have shown that a 150-mg dosage is superior to placebo $^{34}$ for prevention of migraine, with a median reduction of four days with migraine in the last two weeks of therapy compared to the first two weeks. Antidepressants should be considered for prophylaxis of migraine in patients with a coexisting anxiety or mood disorder.

\section{Antihypertensives}

A Cochrane review of 26 studies of propranolol ${ }^{36}$ at any dose versus placebo for migraine prophylaxis involved 668 patients. The relative risk of a 50\% reduction in migraine frequency with propranolol relative to placebo was 1.94 (95\% CI 1.61$2.35, p<0.00001)$. The dropout rate for adverse events was low $(<5 \%)$. The main adverse effects of propranolol are fatigue, and reduction of heart rate and blood pressure.

A meta-analysis of studies comparing the responder rate of propranolol to nadolol for prevention of migraine favoured nadolol (RR 0.60, 95\% CI 0.37-0.97, $p=0.04$ ). ${ }^{36}$ A metaanalysis of studies comparing propranolol to metoprolol did not show a substantial difference between treatments with respect to efficacy. ${ }^{36}$ No significant difference between propranolol and other $\beta$-blockers was reported for adverse events or for dropouts. A single, good-quality crossover trial evaluating candesartan ${ }^{51}$ versus placebo for prophylaxis of migraine in 57 patients showed a responder rate of $40 \%$ during treatment with candesartan compared to $4 \%$ during the placebo 
Table 1: Prophylactic medications for migraine by quality of supporting evidence, clinical impression of efficacy, and adverse effects

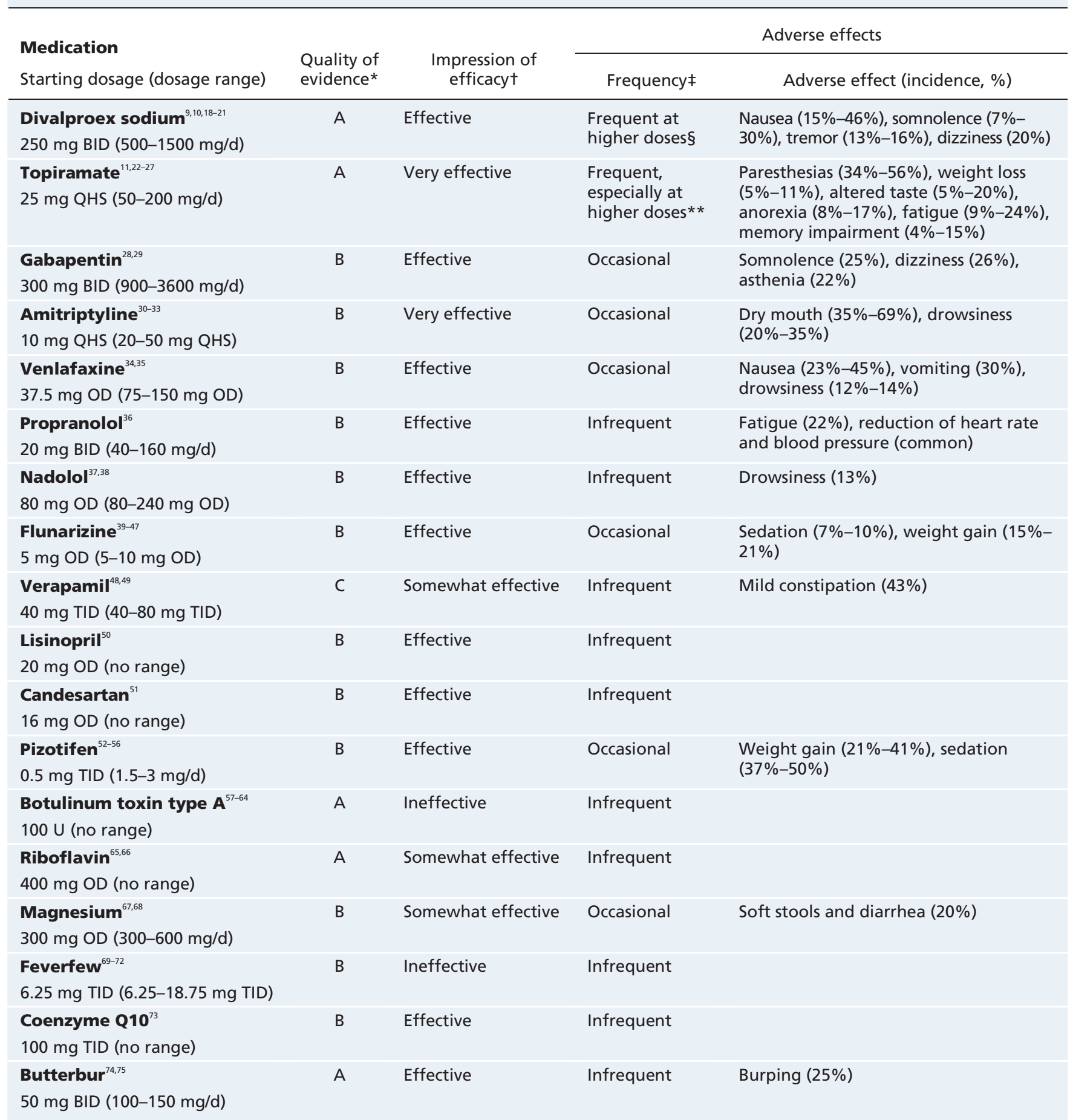

Note: BID = twice daily, OD = once daily, QHS = once daily at bedtime, TID = three times daily.

*Based on the United States Preventive Services Task Force criteria: ${ }^{76}$ A: multiple, well-designed randomized controlled trials yielded a consistent pattern of findings. B: some evidence from randomized controlled trials exists, but scientific support is not optimal (i.e., only one well-designed randomized controlled trial exists, or existing trials have some methodologic limitations, or existing trials yielded inconsistent results). C: randomized controlled trials had major methodological flaws. tBased on clinical experience and results of randomized controlled studies. Ineffective: most people experience no improvement. Somewhat effective: few people experience clinically significant improvement. Effective: some people experience clinically significant improvement. Very effective: most people experience clinically significant improvement.

$¥ B$ ased on results from randomized controlled studies. Infrequent: led to discontinuation of therapy in < $10 \%$ of patients. Occasional: led to discontinuation of therapy in $10 \%-25 \%$ of patients. Frequent: led to discontinuation of therapy in $>25 \%$ of patients.

$\S$ The highest drop-out rate due to adverse effects was $27 \%$ with a dosage of $1500 \mathrm{mg}$.

**Drop-out rates of up to $30 \%$ occurred due to adverse effects, especially with a dosage of $200 \mathrm{mg}$. 
phase $(p<0.001)$. A single good-quality crossover trial of lisinopril ${ }^{50}$ versus placebo for migraine prophylaxis in 60 patients found a $22 \%(95 \%$ CI $11 \%-33 \%)$ reduction in days with migraine during the lisinopril phase compared with the placebo phase. In both trials, adverse effects were no greater in the treatment groups than with placebo. Antihypertensive agents can reasonably be offered for migraine prophylaxis to patients with a history of hypertension or coexisting cardiovascular disease.

\section{Vitamins, minerals and herbal agents}

One good-quality study of riboflavin $400 \mathrm{mg}$ per day versus placebo $^{65}$ for migraine prophylaxis involving 55 patients found an odds ratio of 5.6 (95\% CI 1.64-19.5, $p=0.006$ ) for a $50 \%$ or greater reduction in migraine frequency. Adverse effects were not different between the two groups. A single fair-quality of study of coenzyme Q10 ${ }^{73}$ (300 mg per day) versus placebo involving 42 patients with migraine reported an odds ratio of 5.45 (95\% CI 1.23-24.26, $p=0.03$ ) for a $50 \%$ or greater reduction in migraine frequency relative to placebo. Tolerability was excellent; only one patient treated with coenzyme Q10 withdrew (because of a cutaneous allergy).

The efficacy of butterbur in migraine prevention has been assessed in two good-quality trials. Our meta-analysis of these two studies involving a 50-mg twice-daily dosage of butterbur ${ }^{74,75}$ showed an odds ratio of 2.24 for a $50 \%$ reduction in frequency of migraine episodes (95\% CI 0.64-7.81, $p=$ $0.20)$ compared with placebo. For a $75-\mathrm{mg}$ twice-daily dosage, assessed by one of these studies ${ }^{75}$ the odds ratio for reduction in migraine frequency was 2.16 (95\% CI 1.06-4.38, $p=0.03$ ). Significant differences in the incidence of adverse events between butterbur and placebo were observed for burping only.
One 12-week trial of magnesium citrate $600 \mathrm{mg}$ versus placebo $^{68}$ showed a significantly higher reduction in episode frequency in the final month of treatment relative to baseline in patients treated with magnesium $(-1.51$ episodes) than the placebo group ( -0.58 episodes, $p=0.03)$. Eight $(19 \%)$ of the patients receiving treament with magnesium experienced soft stools or diarrhea, leading to discontinuation of treatment in two patients. Magnesium is the only preventive agent with a category-A pregnancy rating, and is therefore an appropriate choice for prophylaxis in women who are pregnant or trying to conceive. Health Canada recommends a maximum daily dose of magnesium of $350 \mathrm{mg}$ for pregnant women. ${ }^{79}$

\section{Other pharmacologic therapies}

We identified eight studies assessing botulinum toxin type A for the prophylaxis of migraine. Six of these trials ${ }^{57-62}$ did not show an impact of treatment with botulinum toxin type A on frequency of migraine. One study identified a positive result in a subgroup analysis. ${ }^{63}$ Another study did not have interpretable results for effect on migraine frequency. ${ }^{64}$ Existing evidence does not support the efficacy of botulinum toxin type A for the prophylaxis of migraine headache.

Five studies compared pizotifen to placebo for prophylaxis of migraine..$^{52-56}$ These studies could not be combined using meta-analysis, because of substantial clinical heterogeneity. All five studies reported a beneficial effect of pizotifen over placebo for migraine prophylaxis. Ostermann ${ }^{55}$ reported a $50 \%$ responder rate in $43 \%$ of participants during treament with pizotifen, compared with $4 \%$ during placebo treatment in a crossover study. The main adverse effects observed in all of the studies were weight gain and sedation. The highest rate of dropouts owing to adverse effects in the five studies was $14 \%$.

Table 2: Guide to choosing migraine prophylactic drugs

\begin{tabular}{|c|c|c|}
\hline First-line agents & Second-line agents & Third-line agents \\
\hline Propranolol & Gabapentin & Pizotifen \\
\hline \multirow{4}{*}{ Nadolol } & Candesartan & \\
\hline & Lisinopril & \\
\hline & Coenzyme Q10 & \\
\hline & Riboflavin & \\
\hline Special considerations & Appropriate agents & \\
\hline Hypertension or cardiovascular disease & \multicolumn{2}{|c|}{ Propranolol, nadolol, lisinopril, candesartan } \\
\hline Pregnant or trying to conceive & \multicolumn{2}{|l|}{ Magnesium } \\
\hline Obese & \multicolumn{2}{|l|}{ Topiramate } \\
\hline Poor tolerance of medication side effects & \multicolumn{2}{|c|}{ Riboflavin, coenzyme Q10, butterbur, propranolol, lisinopril, candesartan } \\
\hline
\end{tabular}


We found six trials comparing flunarizine to placebo..$^{39-44}$ Because of their substantial clinical heterogeneity, we did not combine these trials statistically. All of them reported a substantial reduction in frequency of migraine with flunarizine 10 mg compared with placebo. The trial by Sorensen ${ }^{43}$ found that the median number of migraines over a four-week period using flunarizine $10 \mathrm{mg}$ was 2.0 , compared with 3.2 during placebo treatment $(p<0.05)$ and 3.5 during the four-week baseline $(p<0.05)$. The most common adverse effects experienced from flunarizine were sedation and weight gain.

\section{How should prophylaxis be initiated?}

The choice between prophylactic treatments is often based on adverse effects. Medications with the fewest adverse effects included propranolol, lisinopril, candesartan, riboflavin, coenzyme Q10 and butterbur. Such medications should be initiated in patients who have a history of poor tolerance to adverse effects of medication. Clinical experience among headache specialists suggests that the antiepileptics, antihypertensives and antidepressants have greater clinical effect than vitamins, minerals and herbal agents (which generally have limited efficacy but very low adverse-effect profiles). Unfortunately, given the limited number of head-to-head trials comparing different prophylactic regimens, evidence-based statements about the relative efficacy of medications cannot be made. Migraine prophylactic medications should be started at a low dosage and gradually increased over several weeks to the target dosage to avoid adverse effects. Patients should be cautioned that an adequate trial of medication takes eight to twelve weeks, and that more than one agent may need to be tried.

\section{When should prophylaxis be stopped?}

Little evidence exists to suggest how long successful migraine prophylaxis should be continued. Recent studies suggest that most patients relapse to some extent after cessation of prophylactic therapy. One study found that $75 \%$ of patients developed increased frequency of migraine episodes when successful prophylaxis was stopped. ${ }^{80}$ Athough the time to relapse was highly variable from patient to patient, it occurred an average of six months after cessation of prophylaxis.

Another study, which randomized patients to placebo or continued topiramate therapy after 26 weeks of topiramate prophylaxis, found that within one month, patients on placebo had deteriorated substantially in terms of migraine frequency compared with those who continued topiramate therapy. ${ }^{8}{ }^{8}$ However, even after 26 weeks on placebo, headache frequency had not increased to the frequency at baseline (i.e., before starting topiramate prophylaxis).

These findings suggest that in many patients migraine prophylactics should be stopped at some point to see if they are still needed. Data from placebo-controlled crossover trials of valproate ${ }^{20}$ and flunarizine ${ }^{43}$ show that, after short-term prophylactic therapy (usually three months), the benefits begin to wane within four weeks of stopping prophylaxis.

Existing guidelines either do not address discontinuation, ${ }^{2}$ or they recommend tapering the prophylactic medication after three to six months, if headaches are well controlled, ${ }^{1}$ or after "several" months. ${ }^{6}$ Given the evidence that the effects of prophylactic medications begin to wane quickly after prophylaxis is stopped, continuing prophylaxis for much longer is prudent for patients with difficult migraine that has caused substantial disability in the past. The ultimate decision depends on the benefit experienced by the patient and whether substantial adverse effects are experienced.

Follow-up every three to six months is important for patients taking prophylaxis, for if the initial benefits of the prophylactic medication eventually disappear, little apparent purpose remains in continuing it. Diary-based documentation of headache frequency can be helpful in making such decisions. Generally, when the decision to stop prophylaxis is made, the dosage of medication is tapered over a two- to four-week period.

\section{When should a patient be referred to a headache specialist?}

The generalist should refer a patient whenever questions about the diagnosis, treatment or response exceed the clinical comfort of dealing with uncertainty. In particular, referral is expected when the phenomenology of the headache does not fit a recognized pattern. Treatment-related dilemmas leading to referral may arise when the patient fails to respond to usual therapy, when medication overuse contributes to the headache burden, when regular use of opiates is considered or occurs, or when patients' comorbidities, intolerance or preferences limit pharmacologic treatment.

\section{Gaps in knowledge}

Many aspects of migraine prophylactic therapy are not well understood. In particular, the mechanisms by which established prophylactic medications reduce frequency of migraine episodes are not known, and this lack of information has hampered development of more effective medications. Because few head-to-head medication-comparison trials have been done, little evidence exists to guide the choice of medication based on relative efficacy. For patients with refractory migraine, no evidence exists to guide decision-making in the use of combinations of prophylactic medications.

\section{The case revisited}

After discussion, the patient was given topiramate $25 \mathrm{mg}$ to take at bedtime, with a plan to increase the dosage by $25 \mathrm{mg}$ every week, up to a total of $100 \mathrm{mg}$ per day. Prophylaxis was indicated because the patient's migraines caused her substantial disability, and her use of triptan was approaching levels that put her at risk for medication overuse headache. Treament with a $\beta$-blocker was contraindicated by her history of asthma. She had no sleep disturbance or mood disorder to support use of a tricyclic, which might have promoted further weight gain. Topiramate, supported by good evidence of efficacy for migraine prophylaxis, might assist her with weight loss. 


\section{This article has been peer reviewed.}

Competing interests: Tamara Pringsheim has received speaker honoraria (less than \$10 000) from Pfizer. Werner Becker has served on advisory boards or has received speaker honoraria or funding for clinical trials research (less than $\$ 10000$ per company) from Merck Frosst Canada, Pfizer, Allergan, Medtronic, Teva, Amplatzer, and Proctor and Gamble. None declared for Jeptha Davenport.

Contributors: Tamara Pringsheim performed the literature searches, reviewed abstracts, graded the methodological quality of the included studies, performed meta-analyses, analyzed and synthesized results, and drafted most of the manuscript. Jeptha Davenport graded the methodological quality of the included studies, analyzed and synthesized results, and drafted portions of the manuscript. Werner Becker reviewed abstracts, analyzed and synthesized results, and wrote selected portions of the manuscript. All of the authors critically reviewed the manuscript for important intellectual content, and all of them approved the final version submitted for publication.

Acknowledgements: The authors thank Dr. Gordon Mackie and Dr. Marek Gawel for their comments on this review.

\section{REFERENCES}

1. International Headache Society. The international classification of headache disorders. 2nd ed. Cephalalgia 2004;24(Suppl 1):1-160.

2. Silberstein SD and the United States Headache Consortium. Practice parameter Evidence-based guidelines for migraine headache (an evidence-based review) Neurology 2000;55:754-62.

3. Members of the European Federation of Neurological Societies task force: Evers S, Afra J, Frese A, et al. EFNS guideline on the drug treatment of migraine - report of an EFNS task force. Eur J Neurol 2006;13:560-72.

4. Zeeberg P, Olesen J, Jensen R. Probable medication-overuse headache: the effect of a 2-month drug-free period. Neurology 2006;66:1894-8.

5. Jelinski SE, Becker WJ, Christie SN, et al. Clinical features and pharmacological treatment of migraine patients referred to headache specialists in Canada. Cephalalgia 2006;26:578-88

6. Pryse-Phillips WEM, Dodick DW, Edmeads JG, et al. Guidelines for the diagnosis and management of migraine in clinical practice. CMAJ 1997;156:1273-87.

7. Lipton RB, Bigal ME, Diamond M, et al.; the AMPP advisory group. Migraine prevalence, disease burden, and the need for preventative therapy. Neurology 2007:68:343-9.

8. Ziegler DK, Hurwitz A, Preskorn S, et al. Propranolol and amitriptyline in prophylaxis of migraine. Pharmacokinetic and therapeutic effects. Arch Neurol 1993; 50:825-30.

9. Mathew NT, Saper JR, Silberstein SD, et al. Migraine prophylaxis with divalproex Arch Neurol 1995;52:281-6.

10. Klapper, J. Divalproex sodium in migraine prophylaxis: a dose-controlled study. Cephalalgia 1997;2:103-8.

11. Brandes JL, Saper JR, Diamond M, et al. Topiramate for migraine prevention. JAMA 2004;291:965-73.

12. Diener H-C, Bussone G, Van Oene JC, et al. TOPMAT-MIG 201 (TOP-Chrome) study group. Topiramate reduces headache days in chronic migraine: a randomized, double-blind, placebo-controlled study. Cephalalgia 2007;27:814-23.

13. Mei D, Ferraro D, Zelano G, et al. Topiramate and triptans revert chronic migraine with medication overuse to episodic migraine. Clin Neuropharmacol 2006;29:269-75.

14. Lipton RB. Tracing transformation. Chronic migraine classification, progression and epidemiology. Neurology 2009;72(Suppl 5):S3-7.

15. Katsarava Z, Schneeweiss S, Kurth T, et al. Incidence and predictors for chronicity of headache in patients with episodic migraine. Neurology 2004;62:788-90.

16. Limmroth V, Biondi $\mathrm{D}$, Pfeil J, et al. Topiramate in patients with episodic migraine reducing the risk for chronic forms of headache. Headache 2007;47:13-21.

17. Ad hoc committee on classification of headache: classification of headache. JAMA 1962;179:717-8

18. Freitag FG, Collins SD, Carlson HA, et al. A randomized trial of divalproex sodium extended release tablets in migraine prophylaxis. Neurology 2002;58:1652-9.

19. Hering R, Kuritzky A. Sodium valproate in the prophylactic treatment of migraine: a double-blind study versus placebo. Cephalalgia 1992;12:81-4.

20. Jensen R, Brinck T, Olesen J. Sodium valproate has a prophylactic effect in migraine without aura: a triple blind, placebo controlled crossover study. Neurology 1994;44:647-51.

21. Shaygannejad V, Janghorbani M, Ghorbani A, et al. Comparison of the effect of topiramate and sodium valproate in migraine prevention: a randomized blinded crossover study. Headache 2006;46:642-8.

22. Diener $\mathrm{H}$, Tfelt-Hansen $\mathrm{P}$, Dahlof $\mathrm{C}$, et al. Topiramate in migraine prophylaxis. $J$ Neurol 2004;251:943-50.

23. Mei D, Capuano A, Vollono C, et al. Topiramate in migraine prophylaxis: a randomized double blind versus placebo study. Neurol Sci 2004;25:245-50.

24. Silberstein SD, Neto W, Schmitt J, et al. Topiramate in migraine prevention. Arch Neurol 2004;61:490-5
25. Storey JR, Calder CS, Hart DE, et al. Topiramate in migraine prevention: a doubleblind, placebo-controlled study. Headache 2001;41:968-75

26. Gupta P, Singh S, Goyal V, et al. Low dose topiramate versus lamotrigine in migraine prophylaxis (the Lotolamp study). Headache 2007;47:402-12.

27. Silberstein SD, Hulihan J, Karim MR, et al. Efficacy and tolerability of topiramate $200 \mathrm{mg} / \mathrm{d}$ in the prevention of migraine with/without aura in adults: a randomized, placebo-controlled, double-blind, 12-week pilot study. Clin Ther 2006;28:1002-11.

28. Mathew NT, Rapoport A, Saper J, et al. Efficacy of gabapentin in migraine prophylaxis. Headache 2001;41:119-28

29. Di Trapani B, Mei D, Marra C, et al. Gabapentin in the prophylaxis of migraine: a double-blind randomized placebo controlled study. Clin Ter 2000;151:145-8.

30. Bank J. A comparative study of amitriptyline and fluvoxamine in migraine prophylaxis. Headache 1994;34:476-8.

31. Couch JR, Hassanein RS. Amitriptyline in migraine prophylaxis. Arch Neurol 1979;36:695-9.

32. Gomersall JD, Stuart A. Amitriptyline in migraine prophylaxis. J Neurol Neurosurg Psychiatry 1973;36:684-90.

33. Ziegler DK, Hurwitz A, Hassanein RS. Migraine prophylaxis. A comparison of propranolol and amitriptyline. Arch Neurol 1987;44:486-9.

34. Ozyalcin SN, Talu GK, Kiziltan E, et al. The efficacy and safety of venlafaxine in the prophylaxis of migraine. Headache 2005;45:144-52.

35. Bulut S, Berilgen MS, Baran A, et al. Venlafaxine versus amitriptyline in the prophylactic treatment of migraine: randomized, double-blind, crossover study. Clin Neurol Neurosurg 2004;107:44-8.

36. Linde K, Rossnagel K. Propranolol for migraine prophylaxis [review]. Cochrane Database Syst Rev 2004;(2):CD003225.

37. Ryan RE Sr., Ryan RE Jr., Sudilovsky A. Nadolol and placebo comparison study in the prophylactic treatment of migraine. Panminerva Med 1982;24:89-94.

38. Freitag FG, Diamond S. Nadolol and placebo comparison study in the prophylactic treatment of migraine. J Am Osteopath Assoc 1984;84:343-7.

39. Al Deeb SM, Biary N, Bahou Y, et al. Flunarizine in migraine: a double-blind placebo-controlled study (in a Saudi population). Headache 1992;32:461-2.

40. Frenken CW, Nuijten ST. Flunarizine, a new preventive approach to migraine. Clin Neurol Neurosurg 1984;86:17-20.

41. Louis P. A double-blind placebo-controlled prophylactic study of flunarizine in migraine. Headache 1981;21:235-9.

42. Mendenopoulos G, Manafi TH, Logothetis I, et al. Flunarizine in the prevention of classical migraine: a placebo-controlled evaluation. Cephalalgia 1985;5:31-7.

43. Sorensen PS, Hansen K, Olesen J. A placebo-controlled, double-blind cross-over trial of flunarizine in common migraine. Cephalalgia 1986;6:7-14.

44. Thomas M, Behari M, Ahuja GK. Flunarizine in migraine prophylaxis: an Indian trial. Headache 1991;31:613-5.

45. Cerbo R, Casacchia M, Formisano R, et al. Flunarizine-pizotifen single dose double blind crossover trial in migraine prophylaxis. Cephalalgia 1986;6:15-8.

46. Louis $\mathrm{P}$, Spierlings EL. Comparison of flunarizine and pizotifen in migraine treatment: a double-blind study. Cephalalgia 1982;2:197-203.

47. Rascol A, Montastruc JL, Rascol O. Flunarizine versus pizotifen: a double blind study in the prophylaxis of migraine. Headache 1986;26:83-5.

48. Markley HG, Cheronis J, Piepho RW. Verapamil in prophylactic therapy of migraine. Neurology 1984:34:973-6.

49. Soloman GD, Steel JG, Spaccavento LJ. Verapamil prophylaxis of migraine: a double-blind, placebo-controlled trial. JAMA 1983;250:2500-2

50. Schrader H, Stovner LJ, Helde G, et al. Prophylactic treatment of migraine with angiotensin converting enzyme inhibitor (lisinopril): randomized, placebo-controlled, crossover study. BMJ 2001;322:1-5.

51. Tronvik E, Stovner LJ, Helde G, et al. Prophylactic treatment of migraine with an angiotensin II receptor blocker. JAMA 2003;289:65-9.

52. Bellevance AJ, Meloche JP. A comparative study of naproxen sodium, pizotyline and placebo in migraine prophylaxis. Headache 1990;30:710-5.

53. Carroll JD, Maclay WP. Pizotifen (BC-105) in migraine prophylaxis. Curr Med Res Opin 1975;3:68-71

54. Lawrence ER, Hossain M, Littlestone W. Sanomigran for migraine prophylaxis; controlled multicentre trial in general practice. Headache 1977;17:109-12.

55. Osterman PO. A comparison between placebo, pizotifen and divascan in migraine prophylaxis. Acta Neurol Scand 1977;56:17-28.

56. Sjaastad O, Stensrud P. Appraisal of BC-105 in migraine prophylaxis. Acta Neurol Scand 1969;45:594-600.

57. Aurora SK, Gawel M, Brandes JL, et al. Botulinum toxin type A prophylactic treatment of episodic migraine: a randomized double blind placebo controlled exploratory study. Headache 2007;47:486-99.

58. Elkind AH, O'Carroll P, Blumenfeld A, et al. A series of three sequential, randomized, controlled studies of repeated treatments with botulinum toxin type A for migraine prophylaxis. J Pain 2006;7:688-96.

59. Evers S, Vollmer-Haase J, Schwaag S, et al. Botulinum toxin A in the prophylactic treatment of migraine - a randomized, double-blind, placebo-controlled study. Cephalalgia 2004;24:838-43.

60. Relja M, Poole AC, Schoenen J, et al. A multicentre, double-blind, randomized, placebo-controlled, parallel group study of multiple treatments of botulinum toxin type A for the prophylaxis of episodic migraine headaches. Cephalalgia 2007;27:492-503.

61. Saper JR, Mathew NT, Loder EW, et al. A double-blind, randomized, placebo-controlled comparison of botulinum toxin type A injection sites and doses in the prevention of episodic migraine. Pain Med 2007;8:478-85.

62. Vo AH, Satori R, Jabbari B, et al. Botulinum toxin type-A in the prevention of migraine: a double-blind controlled trial. Aviat Space Environ Med 2007;78:B113-8. 
63. Silberstein S, Mathew N, Saper J, et al. Botulinum toxin type A as a migraine preventative treatment. Headache 2000;40:445-50.

64. Anand KS, Prasad A, Singh MM, et al. Botulinum toxin type A in prophylactic treatment of migraine. Am J Ther 2006;13:183-7.

65. Schoenen J, Jacquy J, Lenaerts M. Effectiveness of high-dose riboflavin in migraine prophylaxis: a randomized controlled trial. Neurology 1998;50:466-70.

66. Maizels M, Blumenfeld A, Burchette R. A combination of riboflavin, magnesium, and feverfew in migraine prophylaxis: a randomized trial. Headache 2004;44:885-90.

67. Pfaffenrath V, Wessely P, Meyer C, et al. G'bel H, Fischer M. Magnesium in the prophylaxis of migraine - a double-blind, placebo-controlled study. Cephalalgia 1996;16:436-40.

68. Peikert A, Wilimzig C, Kohne-Volland R. Prophylaxis of migraine with oral magnesium: results from a prospective, multicentre, placebo-controlled and double blind randomized study. Cephalalgia 1996;16:257-63.

69. DeWeerdt CJ, Bootsma HPR, Hendriks H. Herbal medicines in migraine prevention: randomized double-blind placebo-controlled crossover trial of a feverfew preparation. Phytomedicine 1996;3:225-30.

70. Diener HC, Pfaffenrath V, Schnitker J, et al. Efficacy and safety of $6.25 \mathrm{mg}$ TID feverfew CO2 extract (MIG-99) in migraine prevention - a randomized, doubleblind, multicentre, placebo controlled study. Cephalalgia 2005;25:1031-41.

71. Murphy JJ, Heptinstall S, Mitchell JR. Randomised double-blind placebo-controlled trial of feverfew in migraine prevention. Lancet 1988;8604:189-92.

72. Pfaffenrath V, Diener H, Fischer M, et al. The efficacy and safety of Tanacetum parthenium (feverfew) in migraine prophylaxis — a double blind, multicentre, randomized placebo-controlled dose-response study. Cephalalgia 2002;22:523-32.

73. Sándor P, Di Clemente L, Coppola G, et al. Efficacy of coenzyme Q10 in migraine prophylaxis: a randomized, controlled trial. Neurology 2005;64:713-5.

74. Lipton RB, Gobel K, Einhaupl KM, et al. Petasites hybridus root (butterbur) is an effective preventative treatment for migraine. Neurology 2004;63:2240-4.

75. Diener HC, Rahlfs VW, Danesch U. The first placebo-controlled trial of a special butterbur root extract for the prevention of migraine: reanalysis of efficacy criteria. Eur Neurol 2004;51:89-97.

76. Harris RP, Helfand MH, Woolf SH, et al. Current methods of the United States Preventive Services Task Force: a review of the process. Am J Prev Med 2001;20(Suppl 3): 21-35.

77. Wyszynski DF, Nambisan M, Surve T, et al. Increased rate of major malformations in offspring exposed to valproate during pregnancy. Neurology 2005;64:961-5.

78. Fraunfelder FW, Fraunfelder FT, Keates EU. Topiramate-associated acute, bilateral, secondary angle closure glaucoma. Ophthalmology 2004;111:109-11.

79. Health Canada. Magnesium. Ottawa (ON): The Department; 2007. Available: www.hc-sc.gc.ca/dhp-mps/prodnatur/applications/licenprod/monograph/mono_magnesium-eng.php\#fn3 (accessed 2009 Sept. 2)

80. Wöeber C, Wöeber-Bingol C, Koch G, et al. Long-term results of migraine prophylaxis with flunarizine and beta-blockers. Cephalalgia 1991;11:251-6.

81. Diener HC, Agosti R, Allais G, et al.; for the TOPMAT-MIG 303 investigator group. Cessation versus continuation of 6-month migraine preventative therapy with topiramate (PROMPT): a randomised, double-blind placebo-controlled trial. Lancet Neurol 2007;6:1054-62.

Correspondence to: Dr. Tamara Pringsheim, Department of Clinical Neurosciences, University of Calgary, Calgary Headache Assessment and Management Program, Foothills Medical Centre, 1403-29th St.NW, Calgary AB T2N 2T9; tmprings@ucalgary.ca 